\title{
Article \\ Analysis and Design of a Wideband Low-Noise Amplifier with Bias and Parasitic Parameters Derived Wide Bandpass Matching Networks
}

\author{
Jinxiang Zhao ${ }^{1,2}$, Feng Wang ${ }^{3}$, Hanchao Yu ${ }^{4}$, Shengli Zhang ${ }^{1,2}\left(\mathbb{0}\right.$, Kuisong Wang ${ }^{1,5}$, Chang Liu ${ }^{1,5}$, Jing Wan ${ }^{1,5}$, \\ Xiaoxin Liang ${ }^{1,5, *}$ and Yuepeng Yan ${ }^{1,5}$
}

check for

updates

Citation: Zhao, J.; Wang, F.; Yu, H.; Zhang, S.; Wang, K.; Liu, C.; Wan, J.; Liang, X.; Yan, Y. Analysis and Design of a Wideband Low-Noise Amplifier with Bias and Parasitic Parameters Derived Wide Bandpass Matching Networks. Electronics 2022, 11, 633. https://doi.org/10.3390/ electronics11040633

Academic Editor: Gaetano Palumbo

Received: 17 January 2022

Accepted: 14 February 2022

Published: 18 February 2022

Publisher's Note: MDPI stays neutral with regard to jurisdictional claims in published maps and institutional affiliations.

Copyright: (c) 2022 by the authors. Licensee MDPI, Basel, Switzerland. This article is an open access article distributed under the terms and conditions of the Creative Commons Attribution (CC BY) license (https:// creativecommons.org/licenses/by/ $4.0 /)$.
1 Communication Center, Institute of Microelectronics, Chinese Academy of Sciences, Beijing 100029, China; zhaojinxiang@ime.ac.cn (J.Z.); zhangshengli@ime.ac.cn (S.Z.); wangkuisong@ime.ac.cn (K.W.); liuchang2021@ime.ac.cn (C.L.); wanjing@ime.ac.cn (J.W.); yanyuepeng@ime.ac.cn (Y.Y.)

2 School of Microelectronics, University of Chinese Academy of Sciences, Beijing 100029, China

3 Bureau of Major Scientific and Technological Tasks, Chinese Academy of Sciences, Beijing 100864, China; wangfeng@cashq.ac.cn

4 Bureau of Frontier Science and Education, Chinese Academy of Sciences, Beijing 100864, China; yuhanchao@cashq.ac.cn

5 Beijing Key Laboratory of New Generation Communication RF Chip Technology, Beijing 100029, China

* Correspondence: liangxiaoxin@ime.ac.cn; Tel.: +86-010-8299-5708

\begin{abstract}
This paper proposes a 110\% relative bandwidth (RBW) low-noise amplifier (LNA) for broadband receivers with flat gain, low noise and high linearity. Bias and parasitic parameters derived wide bandpass (BPDWB) matching networks and a cascode with dual feedbacks are introduced for broadband performance. Matching network design procedures are demonstrated, and results show that the frequency response of the network fits the target impedance well from $1 \mathrm{GHz}$ to $3.5 \mathrm{GHz}$. The proposed BPDWB network improves the design efficiency and enhances the prediction accuracy of impedance matching. The proposed LNA in $0.25 \mu \mathrm{m}$ GaAs pseudomorphic high electron mobility transistor (GaAs pHEMT) technology realizes a minimum NF of $0.45 \mathrm{~dB}$ at $1.6 \mathrm{GHz}$ where the NF is less than $0.55 \mathrm{~dB}$ within the operating frequency band. A flat gain of $22.5-25.2 \mathrm{~dB}$ is achieved with the input voltage standing wave ratio (VSWR) below 1.22 and output VSWR less than 2.5. In addition, the proposed LNA has good linearity where the output third-order intercept point (OIP3) is better than $+31.5 \mathrm{dBm}$, and the output $1 \mathrm{~dB}$ compression point $(\mathrm{OP} 1 \mathrm{~dB})$ is better than $+19 \mathrm{dBm}$ over the wide frequency range.
\end{abstract}

Keywords: low-noise amplifier; wide bandpass matching network; cascode; feedback

\section{Introduction}

Modern wireless communication systems are rapidly developing in the direction of multi-carrier, large-capacity and high-speed information transmission. The demand for high-performance transceivers is increasing [1-3]. As the first active component of the RF front-end, the properties of the LNA, especially bandwidth, noise and linearity, will directly affect the performance of the receiver [4,5]. In response to various RF applications, wideband LNAs handle multiple operating frequencies within a limited chip area, which greatly improves the integration of the RF front-end and plays an important role in many applications such as mobile communications, CATV, satellite navigation, and so on [6-8].

To achieve a balanced performance, the wideband LNA needs to compromise on the strict specifications in a large frequency range, usually several GHz. However, these indicators have a trade-off to each other, which brings challenges to the design of wideband LNAs. Technologies to achieve broad bandwidth with low noise simultaneously are extensively developed and studied [9-14]. In [9], a bandwidth-enhanced Darlington amplifier with shunt feedback is adopted to implement a broadband amplifier with flat gain. However, 
the NF could not be tolerated due to resistance networks at the Darlington structure. In [10], an LNA with a relative bandwidth of $165 \%$ is realized based on the cascade amplifier with resistive feedback. The CS with feedback can achieve a flat broadband bandwidth, but the noise caused by the resistance in feedback is unsatisfactory. Reference [11] explores a bandwidth enhancement technique based on inductive peaking. In [12], a method for designing wideband noise matching is presented.

Compared with semiconductor processes like CMOS and GaN, GaAs has excellent RF performance and cost advantages $[13,14]$. Compared with MOSFET, HBT and other devices, pHEMT devices have better noise performance and thermal stability $[15,16]$. Compared with HEMT, the pHEMT has lower noise and higher electron mobility [17,18]. Therefore, GaAs pHEMT, with excellent RF characteristics and acceptable cost, was selected in this paper.

In order to achieve the goal of wide bandwidth and low noise simultaneously, this paper proposed a wideband LNA using BPDWB matching networks and cascode topology with dual feedbacks. The proposed BPDWB network incorporates the $\mathrm{L} / \mathrm{C}$ in the bias circuit and non-ideal parasitic elements into the design of the matching network, which can realize broadband matching and reduce the negative impact of parasitic components on the circuit concurrently.

The paper is organized as follows: Section 2 introduces the design method of the wideband LNA in detail, including the analysis of the cascode amplifier with dual feedbacks and the design process of the BPDWB network. Section 3 presents the simulation and measurement performance of the proposed LNA and compares it with other published works. Finally, conclusions are given in Section 4.

\section{Proposed LNA Design}

Compared with a narrowband amplifier, a wideband amplifier has the characteristics of large impedance change. To achieve wideband operation, additional circuitry is required. Typically, this means that additional lumped components need to be added to improve the impedance matching of the amplifier to support wideband operation, feedback configuration needs to be added to widen the operable frequency range, or the narrowband amplifier needs to be cascaded [19-21]. In this paper, dual feedbacks and bandpass matching networks are combined to expand the bandwidth of LNA and to select the input signal simultaneously. Figure 1 shows the block diagram of the proposed wideband LNA module. The core of LNA is a cascode topology with dual feedbacks, and the input-output matching is a bandpass network using BPDWB, respectively.

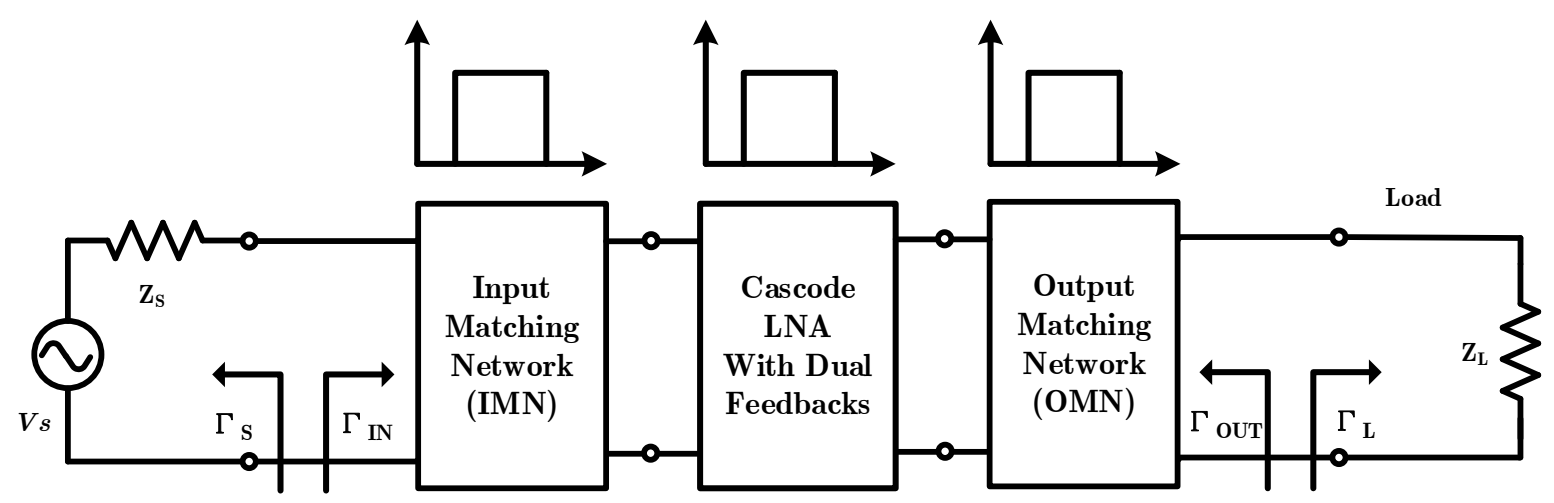

Figure 1. The block diagram of the proposed wideband LNA module.

\subsection{Cascode with Dual Feedbacks}

Linearity and gain are two opposing poles that are difficult to balance, and if we try to improve one, it automatically reduces the other [22]. In this paper, this problem is alleviated by the cascode with feedbacks. The gain of LNA is improved by the stacking of CS and CG, where the Miller effect of CS is cancelled by CG. The linearity is enhanced at the cost 
of gain by introducing feedback [23]. In short, this approach allows a good compromise between gain and linearity.

In this paper, dual feedbacks (series inductance $L_{S}$, parallel resistance $R_{f}$ and capacitance $C_{f}$ ) are connected to the cascode in Figure 2 to achieve high, flat gain and improved linearity.

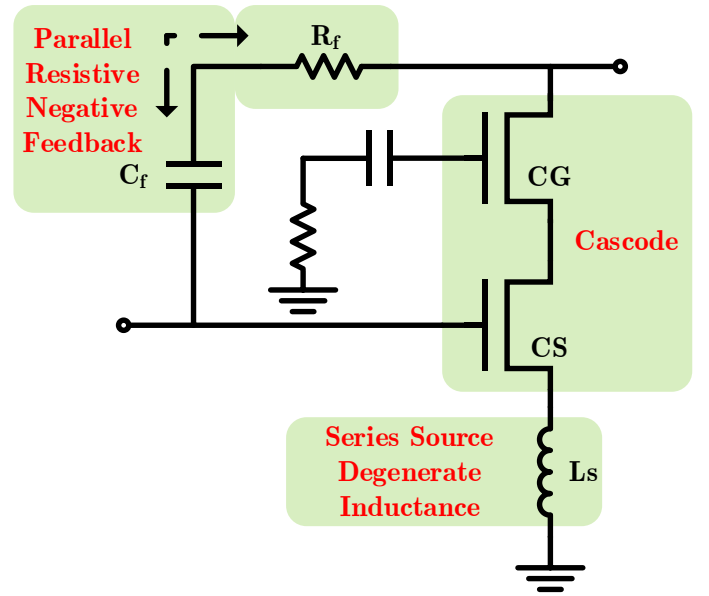

(a)

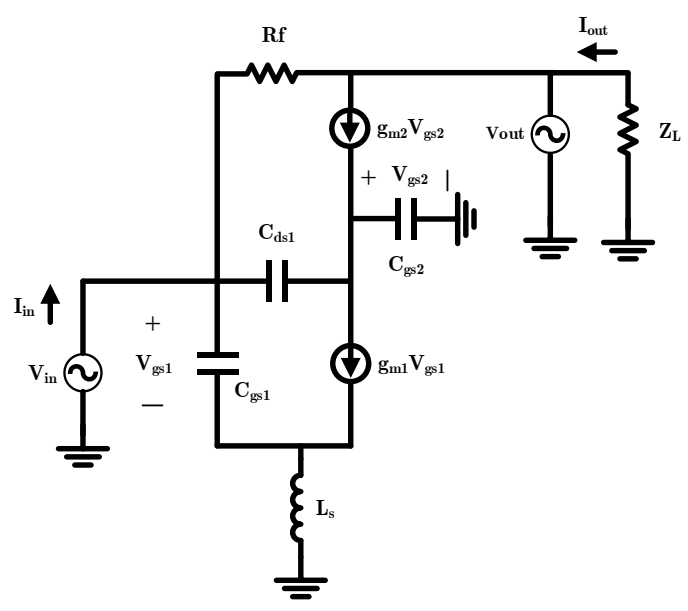

(b)

Figure 2. (a) Cascode with dual feedbacks; (b) The small-signal equivalent model of (a).

For the series inductor $L_{S}$ at the source terminal of $C_{S}$, a real impedance is introduced to help meet the requirement of input matching and noise matching. In theory, simultaneous noise and input impedance matching can be attained. And there is no impact on the noise performance in the case of ideal inductance [23]. However, due to the non-ideality associated with $L_{S}$, additional noise will be introduced to the amplifier. It is necessary to select a suitable value of $L_{S}$ at an acceptable range. When small enough, $L_{S}$ is often replaced by microstrip lines.

For the parallel negative feedback network between the drain terminal of CG and the gate terminal of CS, it couples the AC signal from the output to the input through the feedback capacitor $C_{f}$. The depth of feedback is adjusted by resistor $R_{f}$. Since the feedback resistor will also introduce a noise source, the value of the feedback resistor needs to be chosen properly.

\subsection{Bias and Parasitic Parameters Derived Wide Bandpass Matching Network}

For the structure in Figure $2 b$, the input and output impedance can be equivalent to the form of the capacitance and reactance element in parallel, which exhibits capacitive properties at high frequency. For this case, bandpass matching can be used to achieve ideal matching where ideal bandpass filters can be realized by infinite RLC passive components theoretically [24], as shown in Figure 3, divided into basic units. However, the more components used in the circuit design, the more noise and parasitic are introduced. Several common implementations of bandpass matching circuits are shown below.

To achieve the goal of a bandpass matching network without too many lumped components and improve the design accuracy of the matching network, this paper proposes a bandpass filter matching circuit derived from the bias and parasitic parameters named the BPDWB network. A combination of the bias and parasitic elements is used to compose the basic form of the bandpass filter marked in red in Figure 3. After that, the integrated matching network design is completed with the help of the Smith Chart according to the actual needs of impedance transformation. Figure 4 show two possible input and output matching circuits based on BPDWB. 


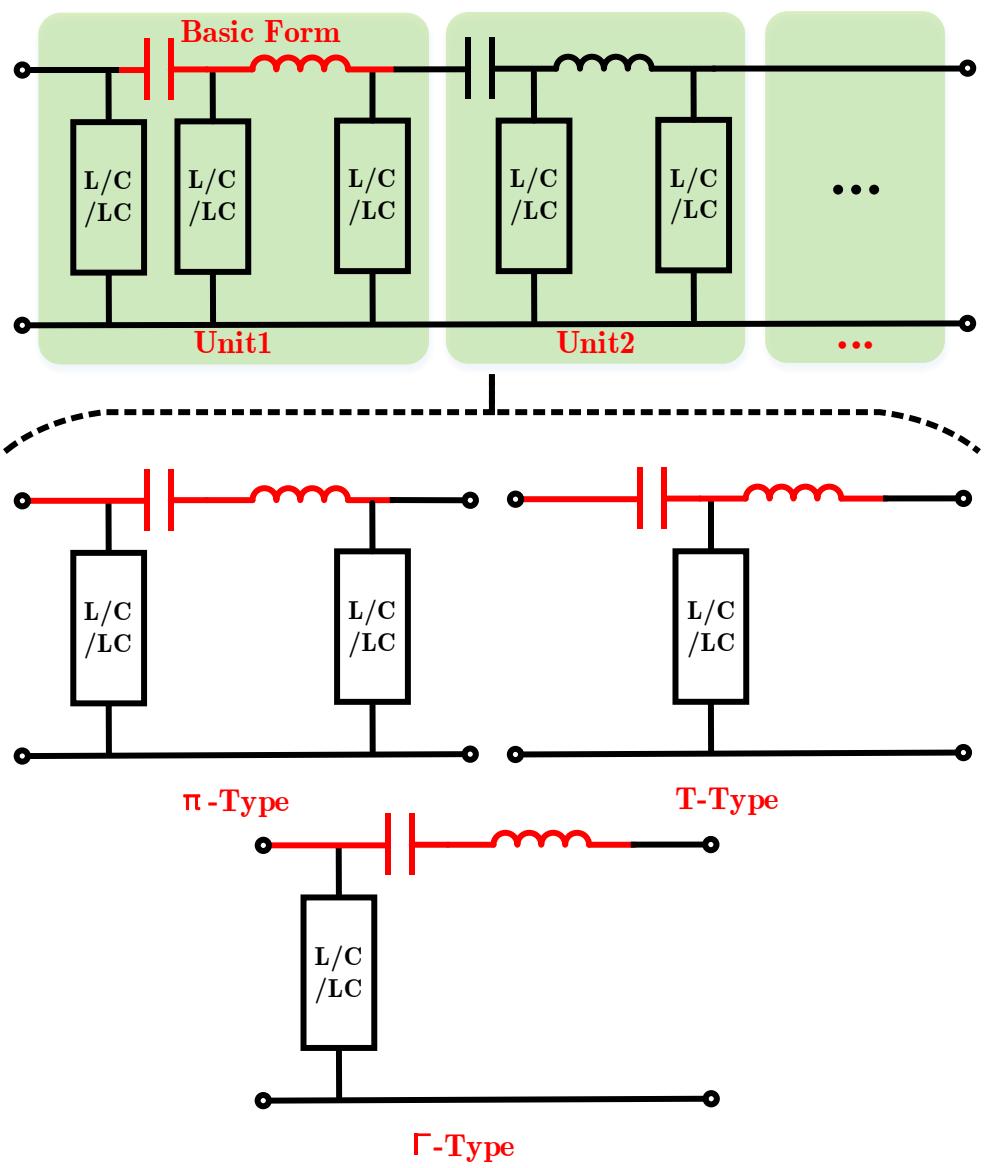

Figure 3. The composition of the bandpass filter.

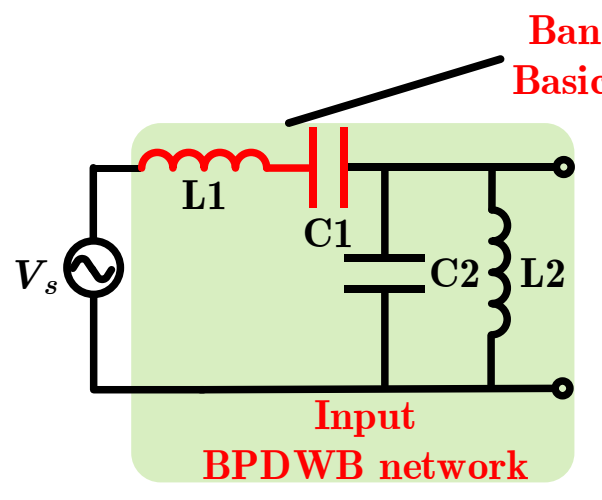

(a)

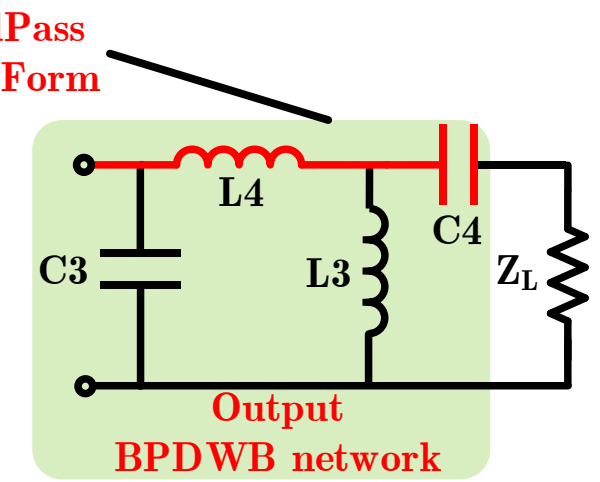

(b)

Figure 4. (a) The proposed input matching network based on BPDWB; (b) output matching network based on BPDWB.

For the input matching network in Figure $4 \mathrm{a}$, it is composed of a series inductor $L_{1}$ and capacitor $C_{1}$ with a parallel capacitor $C_{2}$ and inductor $L_{2} . C_{1}$ can serve as the input DC block, and $L_{2}$ as the gate choke inductor. Bonding wire is incorporated into $L_{1}$. The model of the bonding wire is shown in Figure 5. Its parasitic resistance is usually ignored at RF because it is made of the gold. The capacitor $C_{2}$ includes the parasitic capacitance at the input such as the PAD and ESD. The output network shown in Figure $4 \mathrm{~b}$ is composed of $L_{4}, C_{4}$ in series and $C_{3}, L_{3}$ in parallel. $L_{3}$ can be used as a choke inductor and $C_{4}$ as the output DC block capacitor. $C_{3}$ includes the parasitic capacitance at the output. $C_{2}$ and $C_{3}$ in parallel expand the reactance adjustment range of the matching network so as to fit the frequency response target better. 


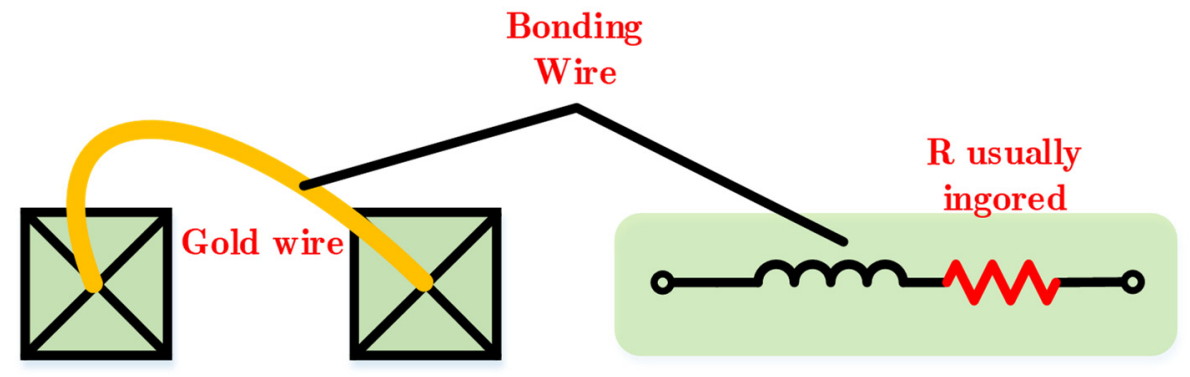

Figure 5. The model of the bonding wire.

Once the structure is determined, the frequency response of the network can be determined by analyzing the transmission characteristics. Regarding the network as a two-port network composed of internal unit circuits, the characteristics of the network can be described by the ABCD matrix.

According to the definition of the ABCD matrix, the input network in Figure 4a can be expressed as

$$
\begin{aligned}
& {\left[\begin{array}{ll}
A & B \\
C & D
\end{array}\right]_{I M N}=\left[\begin{array}{cc}
1 & j \omega L_{1} \\
0 & 1
\end{array}\right]\left[\begin{array}{cc}
1 & 0 \\
j \omega C_{2} & 1
\end{array}\right]\left[\begin{array}{cc}
1 & \frac{1}{j \omega C_{1}} \\
0 & 1
\end{array}\right]\left[\begin{array}{cc}
1 & 0 \\
\frac{1}{j \omega L_{2}} & 1
\end{array}\right]} \\
& =\left[\begin{array}{cc}
1+\frac{C_{2}}{C_{1}}-\omega^{2} C_{2} L_{1}-\frac{1}{\omega^{2} C_{1} L_{2}}+\frac{L_{1}}{L_{2}} & \frac{1}{j \omega C_{1}}+j \omega L_{1} \\
j \omega C_{2}+\frac{1}{j \omega L_{2}} & 1
\end{array}\right]
\end{aligned}
$$

The A parameter of the input network can be obtained as

$$
A_{I M N}=1+\frac{C_{2}}{C_{1}}-\omega^{2} C_{2} L_{1}-\frac{1}{\omega^{2} C_{1} L_{2}}+\frac{L_{1}}{L_{2}}
$$

Therefore, the transfer function of the input matching network can be obtained as

$$
S_{21}(\omega)_{I M N}=\frac{2}{A_{I M N}}=\frac{2}{1+\frac{C_{2}}{C_{1}}-\omega^{2} C_{2} L_{1}-\frac{1}{\omega^{2} C_{1} L_{2}}+\frac{L_{1}}{L_{2}}}
$$

Similarly, the ABCD matrix of the output network in Figure $4 \mathrm{~b}$ is

$$
\begin{aligned}
& {\left[\begin{array}{ll}
A & B \\
C & D
\end{array}\right]_{O M N}=\left[\begin{array}{cc}
1 & \frac{1}{j \omega C_{4}} \\
0 & 1
\end{array}\right]\left[\begin{array}{cc}
1 & 0 \\
\frac{1}{j \omega L_{3}} & 1
\end{array}\right]\left[\begin{array}{cc}
1 & j \omega L_{4} \\
0 & 1
\end{array}\right]\left[\begin{array}{cc}
1 & 0 \\
j \omega C_{3} & 1
\end{array}\right]} \\
& =\left[\begin{array}{cc}
1-\frac{1}{\omega^{2} C_{4} L_{3}}-\omega^{2} C_{3} L_{4}+\frac{C_{3} L_{4}}{C_{4} L_{3}}+\frac{C_{3}}{C_{4}} & j \omega L_{4}+\frac{L_{4}}{j \omega C_{4} L_{3}}+\frac{1}{j \omega C_{4}} \\
\frac{1}{j \omega L_{3}}+\frac{j \omega C_{3} L_{4}}{L_{3}}+j \omega C_{3} & 1+\frac{L_{4}}{L_{3}}
\end{array}\right]
\end{aligned}
$$

The A parameter of the output network can be obtained as

$$
A_{O M N}=1-\frac{1}{\omega^{2} C_{4} L_{3}}-\omega^{2} C_{3} L_{4}+\frac{C_{3} L_{4}}{C_{4} L_{3}}+\frac{C_{3}}{C_{4}}
$$

The transfer function of the output matching network is

$$
S_{21}(\omega)_{O M N}=\frac{2}{A}=\frac{2}{1-\frac{1}{\omega^{2} C_{4} L_{3}}-\omega^{2} C_{3} L_{4}+\frac{C_{3} L_{4}}{C_{4} L_{3}}+\frac{C_{3}}{C_{4}}}
$$

From Equations (3) and (6), it can be seen that the transfer characteristics of matching circuits are affected by constituent units. The target function can be obtained by tuning the value of components which indicates that the BPDWB network possesses strong tunable performance. 


\subsection{Design and Implementation of the Wideband Low-Noise Amplifier}

Composed of the cascode with dual feedbacks and BPDWB matching networks, the complete circuit of the proposed LNA is shown in Figure 6. LNA is designed with a single positive voltage supply of $5 \mathrm{~V}$. Additionally, the size of the CS and CG transistor is $8 \times 75 \mu \mathrm{m}$ and $8 \times 90 \mu \mathrm{m}$. The CS and CG is biased at VGS $=0.62 \mathrm{~V} @ \mathrm{VDS}=1.3 \mathrm{~V}$, $\mathrm{VGS}=0.52 \mathrm{~V} @ \mathrm{VDS}=3.6 \mathrm{~V}$, respectively.

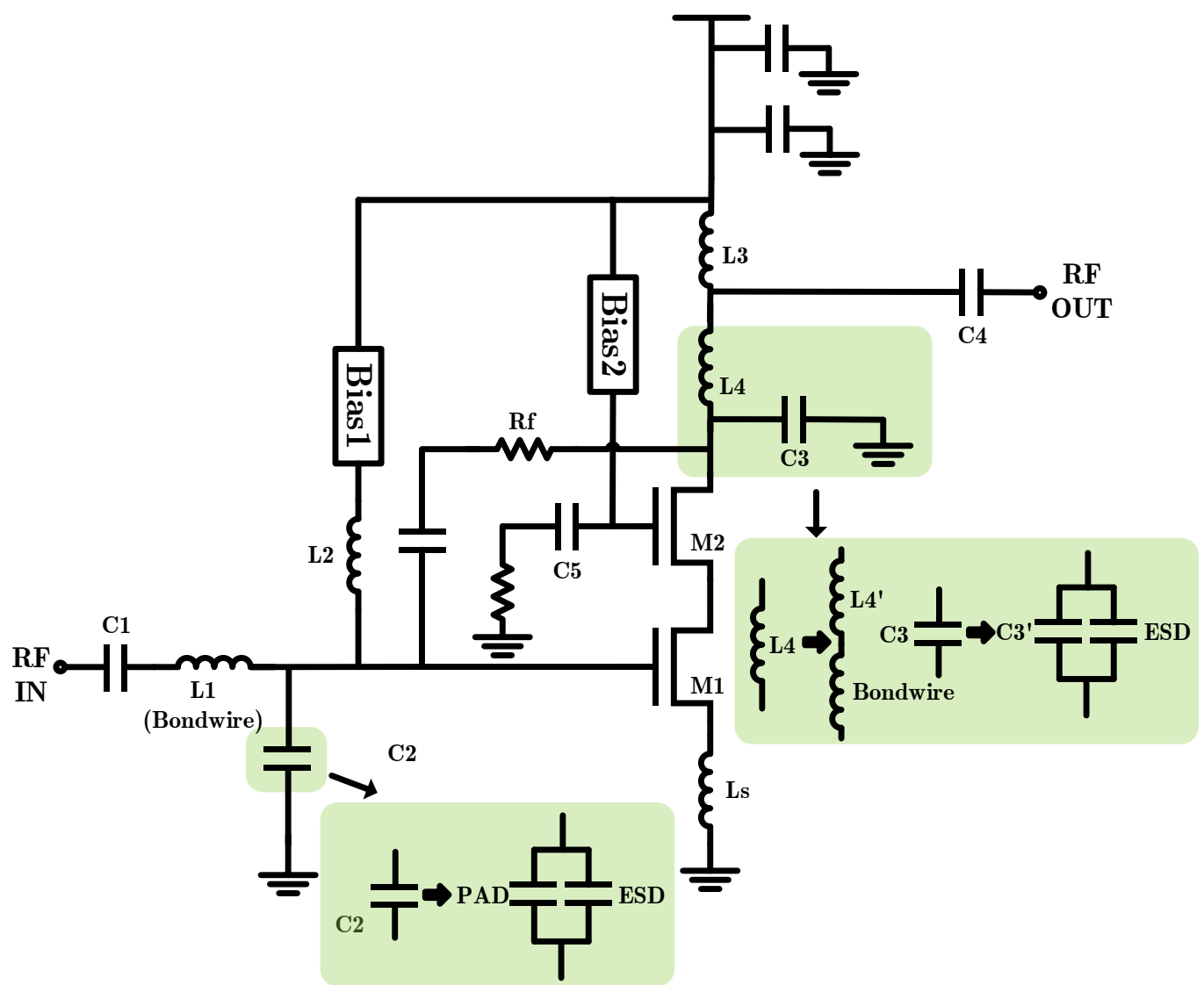

Figure 6. The complete circuit of the proposed LNA.

For expressions (3) and (6), we simplify the analysis by considering the actual situation of the circuit. Since the relation in expressions (7) is:

$$
L_{1}<<L_{2} ; C_{2}<<C_{1}
$$

The following items in Equation (3) can be ignored:

$$
\frac{C_{2}}{C_{1}} \approx 0 ; \frac{L_{1}}{L_{2}} \approx 0
$$

Thus, Equation (3) can be simplified as

$$
S_{21}^{\prime}(\omega)_{I M N} \approx \frac{2}{1-\omega^{2} C_{2} L_{1}-\frac{1}{\omega^{2} C_{1} L_{2}}}=\frac{2 \omega^{2} C_{1} L_{2}}{\omega^{2} C_{1} L_{2}-\omega^{4} C_{1} C_{2} L_{1} L_{2}-1}
$$

It can be found that expression (9) is mainly determined by $C_{1}-L_{2}$ on a numerator at low frequency. Therefore, the target transmission characteristics can be achieved by adjusting the value of $C_{1}-L_{2}$ accompanied by tuning $C_{2}$ and $L_{1}$. 
As frequency increases, Equation (9) can be abbreviated as

$$
S^{\prime \prime}{ }_{21}(\omega)_{I M N} \approx \frac{2 \omega^{2} C_{1} L_{2}}{\omega^{2} C_{1} L_{2}-\omega^{4} C_{1} C_{2} L_{1} L_{2}}=\frac{2}{1-\omega^{2} C_{2} L_{1}}
$$

In Equation (10), the transmission characteristic is mainly affected by the parasitic parameters $L_{1}-C_{2}$. It shows that the parasitic parameters begin to take effect at high frequencies and deteriorate the transmission performance of the network severely. Therefore, the high cut-off frequency of the matching network can be tuned by controlling the value of parasitic parameters, such as the length of the bond wire.

In addition to the $S_{21}$ transmission, noise is also an important index that we should consider synchronously.

For noise figures, we know that

$$
F=F_{M I N}+\frac{G_{n}}{R_{S}}\left|Z_{s}-Z_{S O P T}\right|^{2}
$$

where $F_{M I N}$ is the minimum noise figure, $G_{n}$ is the noise admittance, $R_{s}$ is the real part of complex source impedance and $Z_{S O P T}$ is the optimum source impedance.

As the frequency increases, the $C_{g d}$ of M1 is considered. According to Miller equivalence, $C_{g d}$ and $R_{f}$ can be equivalent to the equivalent capacitance and resistance to ground at the input, that is, to contribute to $C_{2}$ and introduce an equivalent noise source $R_{f M}$.

$$
R_{f M}=R_{f} /\left(1-A_{v}\right)
$$

where $A_{V}$ is the open-loop voltage gain of the amplifier. According to Formula (12), we know that the greater $R_{f}$, the greater $R_{f M}$.

$C_{2}$ affects the $G_{n}, R_{S}$ and $Z_{S}-Z_{S O P T}$, thereby deteriorating the NF. Fortunately, we can try to improve this situation by feedbacks. For $R_{f M}$, the greater its value, the smaller the impact on noise. Therefore, it is necessary to meet the noise requirements by adjusting $L_{s}$ and selecting an appropriate $R_{f}$. Simulation finds that when $R_{f}$ is greater than $2000 \Omega$, the effect on noise is no longer obvious. Finally, $R_{f}=2500 \Omega$ was chosen. On this basis, according to the transmission characteristics and acceptable noise performance, we finally selected $L_{S} \approx 0.1 \mathrm{nH}$.

At the output, the capacitance of the DC blocking capacitor $C_{4}$ is large enough to make the following items close to zero

$$
\frac{C_{3} L_{2}}{C_{4} L_{3}} \approx 0 ; \frac{C_{3}}{C_{4}} \approx 0
$$

Therefore, Equation (6) can be abbreviated as

$$
S_{21}^{\prime}(\omega)_{O M N} \approx \frac{2}{1-\frac{1}{\omega^{2} C_{4} L_{3}}-\omega^{2} C_{3} L_{4}}=\frac{2 \omega^{2} C_{4} L_{3}}{\omega^{2} C_{4} L_{3}-\omega^{4} C_{3} C_{4} L_{3} L_{4}-1}
$$

At low frequencies, the response of the matching network in Equation (14) is mainly affected by $C_{4}-L_{3}$. At high frequencies, the transfer function can be expressed as Equation (15) which is mainly affected by $C_{3}-L_{4}$, especially the high cut-off frequency.

$$
S_{21}^{\prime \prime}(\omega)_{O M N} \approx \frac{2}{1-\omega^{2} C_{3} L_{4}}
$$

Therefore, the output network can keep constant within the target bandwidth by controlling the tuning $C_{3}-C_{4}-L_{3}-L_{4}$, especially $C_{3}-L_{4}$, because high-frequency characteristics are paid special attention to in the design of LNA. In addition, the influence of the output impedance on linearity such as OIP3, should be taken into account in the design of the LNA. According to the two-tone load-pull results, the impedance point for the maximum OIP3 
at frequency $=2.2 \mathrm{GHz}$ is $\mathrm{Z}=60.6+\mathrm{j} \times 12.6 \Omega$. In order to select the appropriate value of $C_{3}-L_{4}$ to meet the requirements of output matching and linearity, different combinations of $C_{3}-L_{4}$ are scanned, and the corresponding results are shown in Figure 7 . According to the results, the inductance $L_{4}=2.5 \mathrm{nH}$ and the capacitance $C_{3}=300 \mathrm{fF}$ are selected, which can meet the requirements of bandwidth, reflection coefficient and linearity at the same time.

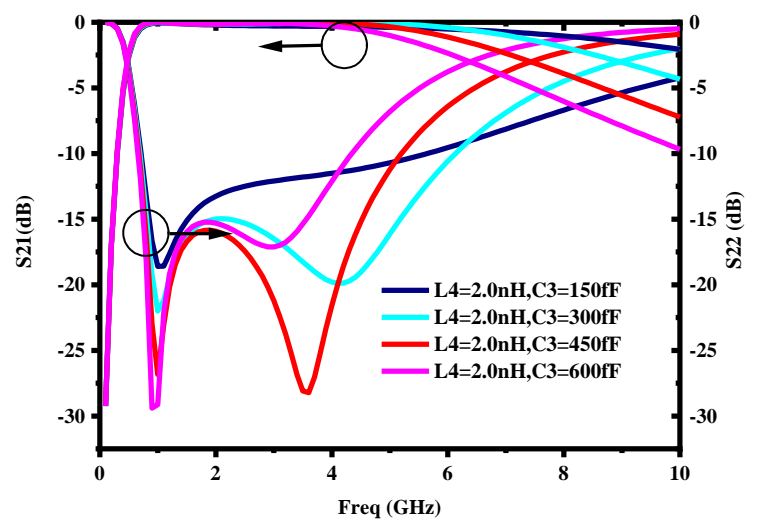

(a)

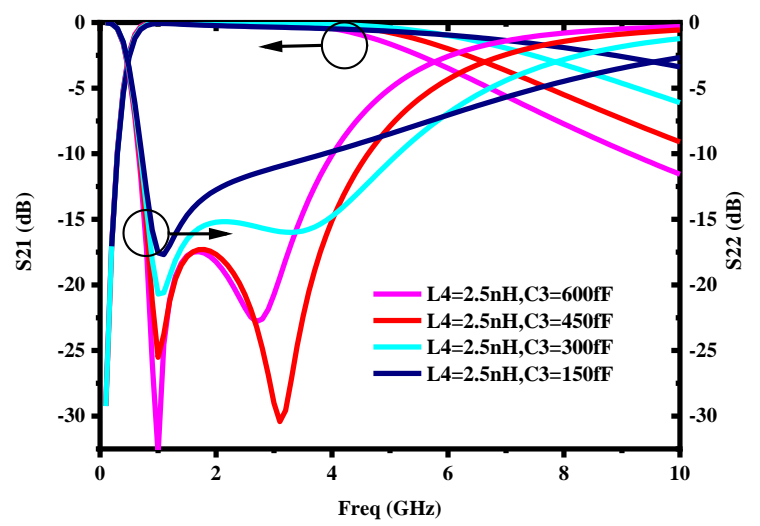

(c)

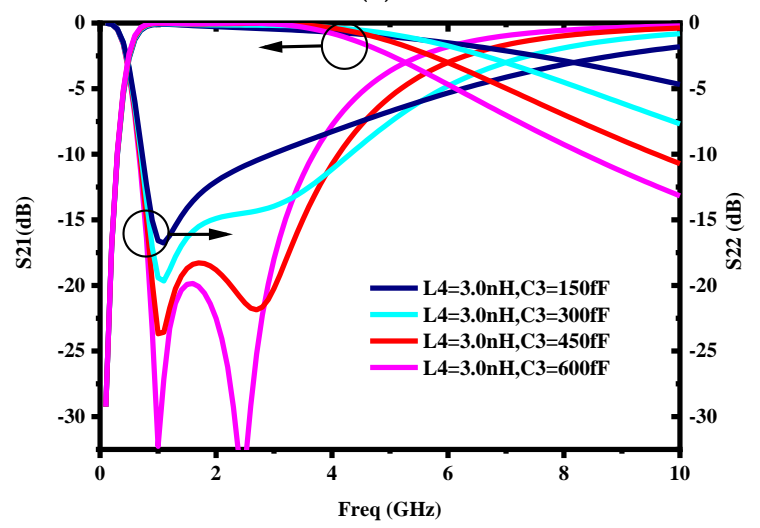

(e)

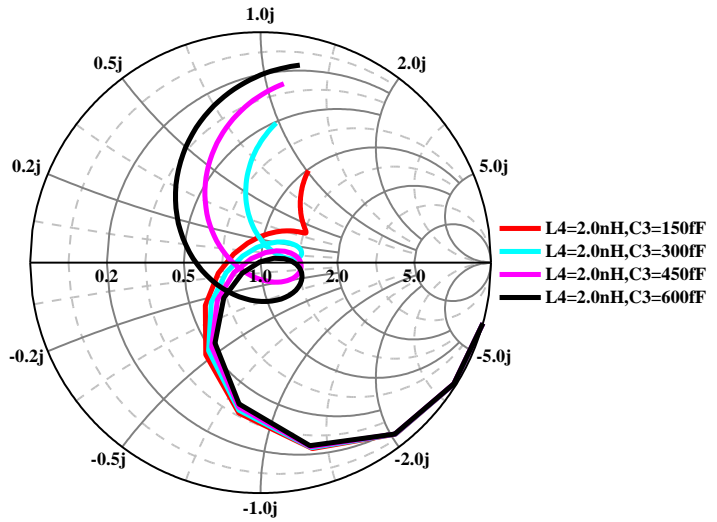

(b)

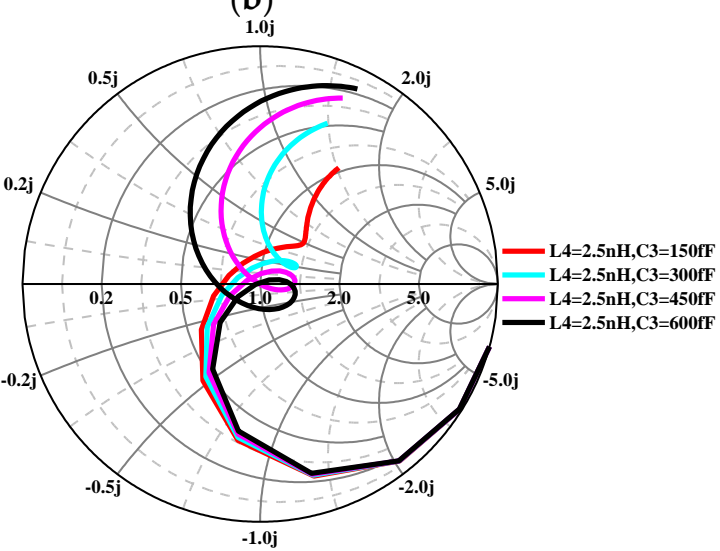

(d)

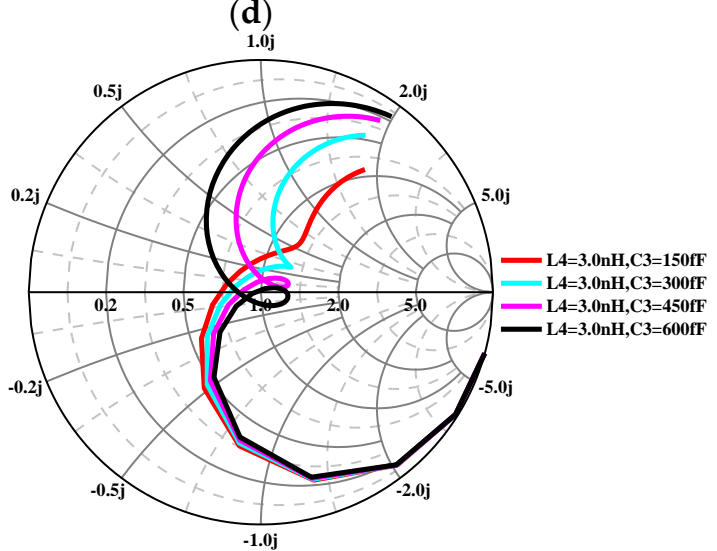

(f)

Figure 7. Performance of different combinations of capacitor $C_{3}$ and inductance $L_{4}$ : (a) S-Parameters for $L_{4}=2 \mathrm{nH}$; (b) Impedance trace of (a); (c) S-Parameters for $L_{4}=2.5 \mathrm{nH}$; (d) Impedance trace of (c); (e) S-Parameters for $L_{4}=3 \mathrm{nH}$; (f) Impedance trace of (e).

Finally, according to the compromise of each index, the values of each component of the proposed LNA are shown in Table 1. 
Table 1. Values of components in circuit.

\begin{tabular}{ccc}
\hline Device & Value & Uint \\
\hline M1 & $8 \times 75$ & $\mu \mathrm{m}$ \\
M2 & $8 \times 90$ & $\mu \mathrm{m}$ \\
$C_{1}$ & 56 & $\mathrm{pF}$ \\
$C_{2}$ & 5.7 & $\mathrm{pF}$ \\
$C_{3}$ & 300 & $\mathrm{pF}$ \\
$C_{4}$ & 6.6 & $\mathrm{pF}$ \\
$C_{5}$ & 2.4 & $\mathrm{pF}$ \\
$L_{1}$ & 0.75 & $\mathrm{nH}$ \\
$L_{2}$ & 9.5 & $\mathrm{nH}$ \\
$L_{3}$ & 9.5 & $\mathrm{nH}$ \\
$L_{4}$ & 2.5 & $\mathrm{nH}$ \\
$L_{s}$ & 0.1 & $\mathrm{nH}$ \\
$R_{f}$ & 2500 & $\Omega$ \\
\hline
\end{tabular}

\section{Measurement Results}

The die and assembly diagram of the chip is shown in Figure 8. The realized LNA is characterized by both linear and non-linear measurements.

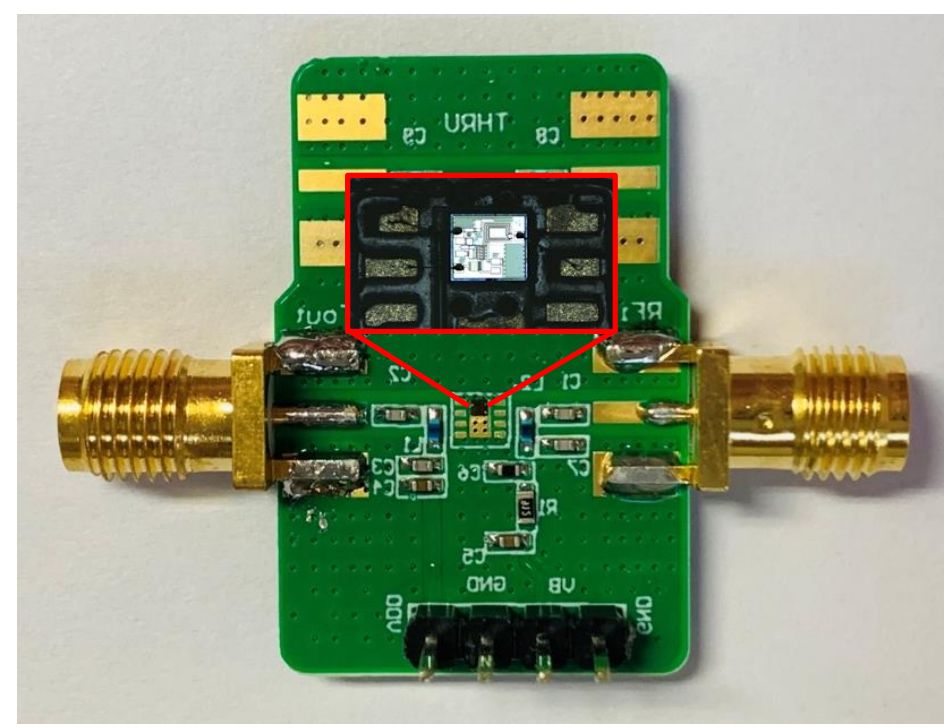

Figure 8. The assembly diagram of the proposed LNA complete circuit.

To define small-signal behavior, LNA scattering parameters were measured with an Agilent N5230A vector network analyzer. The input/output port of the LNA was designed to be $50 \Omega$. A PCB test board was developed to simulate practical scenarios, and bond wires were used to connect to the PCB board at each port on the chip. Figure 9 show the measured scattering parameters of the LNA, together with the simulated ones.

From Figure 9, a peak gain of $25.8 \mathrm{~dB}$ was measured at $1.2 \mathrm{GHz}$, which is $1.4 \mathrm{~dB}$ lower than the simulated result. Measured S11 and S22 was lower than $-20 \mathrm{~dB}$ and $-7 \mathrm{~dB}$ from $1 \mathrm{GHz}$ to $3.5 \mathrm{GHz}$. As far as S11 and S22 are concerned, the difference between simulation and measurement can be ascribed to the input and output connector and to the coupling parasitic between the devices. The $\mathrm{S} 12$ data is lower than $-30 \mathrm{~dB}$ in the frequency band, indicating that the amplifier had a high level of reverse isolation making the LNA unconditionally stable. Measured scattering parameters demonstrated that the BPDWB network had good prediction accuracy for RF LNA.

For the noise performance, the NF measurement was completed by an Agilent N8975A noise figure meter. A low noise figure (NF) of $0.5 \mathrm{~dB}$ (with a ripple of $0.05 \mathrm{~dB}$ ) was registered in the range from $1 \mathrm{GHz}$ to $3.5 \mathrm{GHz}$, shown in Figure 10. Compared with simulation data, 
the measured NF was about $0.1 \mathrm{~dB}$ higher. This was caused by the difference between the device and model. And the simulation could not simulate the real conditions of the working device absolutely.

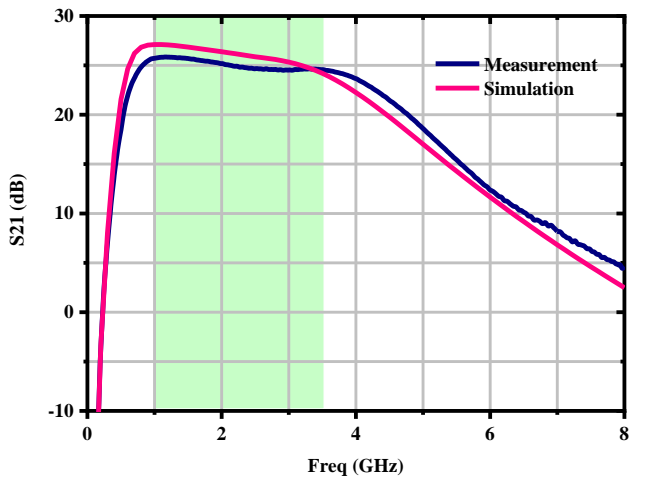

(a)

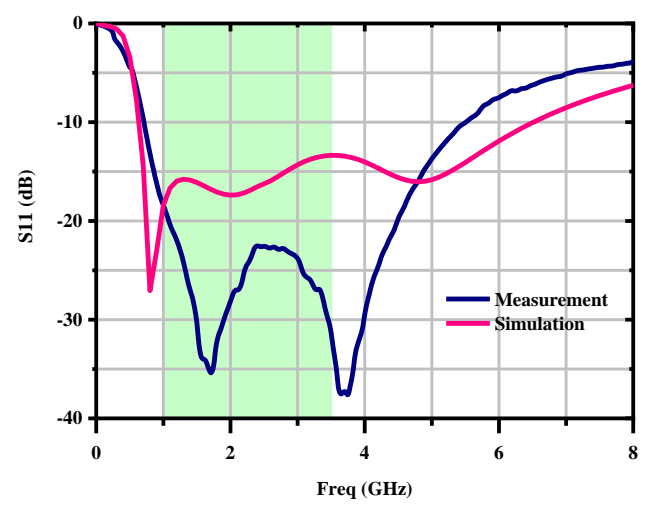

(c)

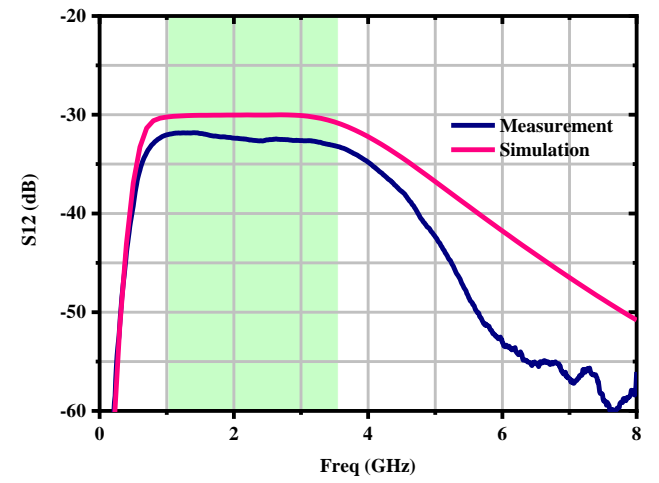

(b)

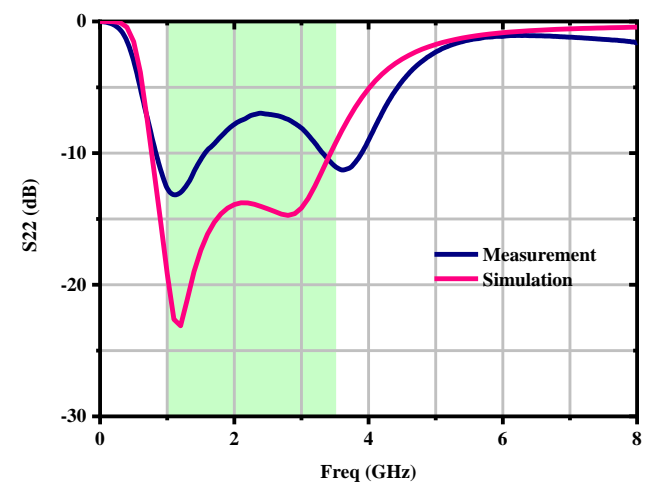

(d)

Figure 9. Scattering parameters of the proposed LNA (a) S21; (b) S12; (c) S11; (d) S22.

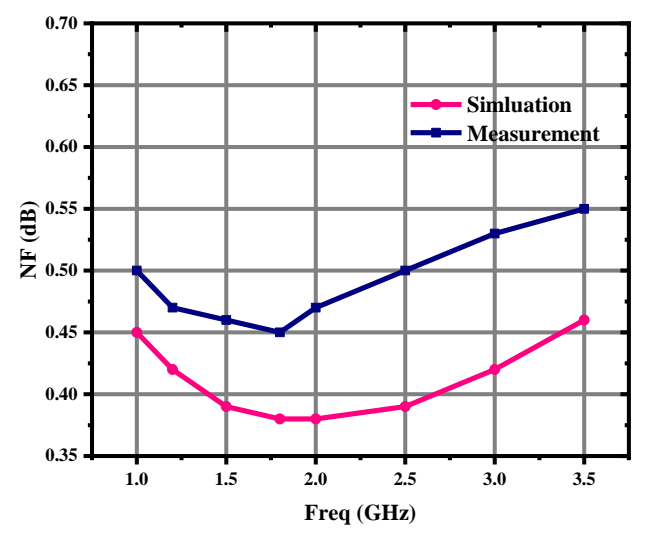

Figure 10. NF performance in the frequency range $1 \mathrm{GHz}-3.5 \mathrm{GHz}$.

To define large-signal behavior, the linearity measurements were performed for the LNA. Figure 11 present the measured OP1dB and OIP3 results with simulated data according to the single-tone output power test and two-tone third-order intermodulation test. The LNA processes the OP1dB better than $+19 \mathrm{dBm}$ and OIP3 greater than $+31.5 \mathrm{dBm}$ by 3.5 GHz. Due to transistor large-signal model errors, the measured linearity data is about $2 \mathrm{~dB}$ lower than the simulated results. 


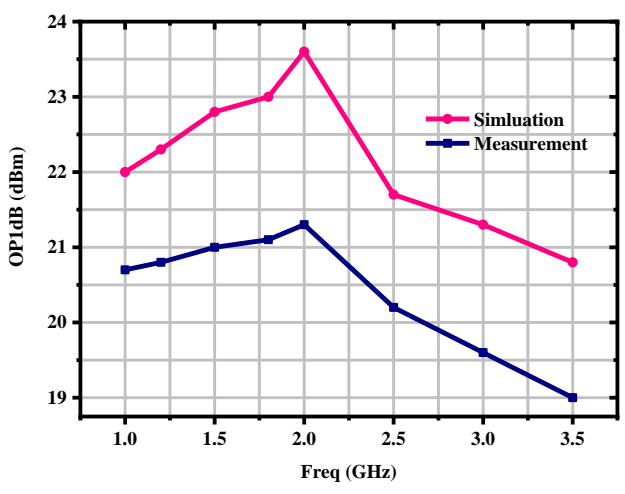

(a)

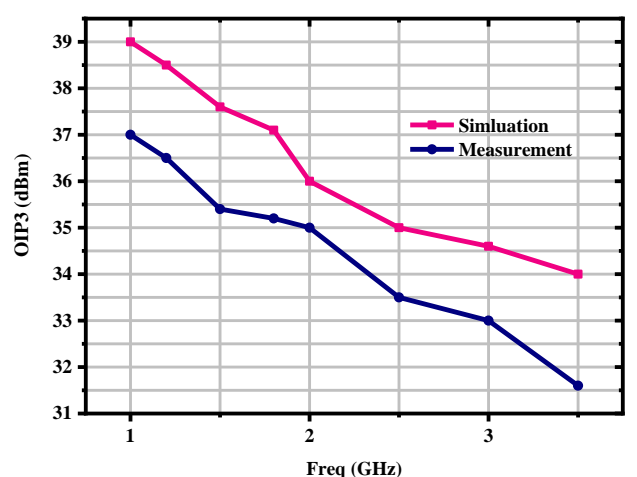

(b)

Figure 11. Linearity performance in the frequency range $1 \mathrm{GHz}-3.5 \mathrm{GHz}(\mathbf{a}) \mathrm{OP} 1 \mathrm{~dB}$; (b) OIP3.

Table 2 present a comprehensive summary of the performance of the designed wideband LNA and a comparison of the latest works of other published LNAs over the L to S-band.

Table 2. Performance summary and comparison.

\begin{tabular}{|c|c|c|c|c|c|c|c|c|c|c|}
\hline Ref. & Process & Architecture/Model & $\begin{array}{l}\text { Bandwidth } \\
\text { (GHz) }\end{array}$ & Gain $(\mathrm{dB})$ & NF (dB) & S11 (dB) & $\begin{array}{c}\text { IIP3 } \\
(\mathrm{dBm})\end{array}$ & $\begin{array}{l}\text { IP1dB } \\
(\mathrm{dBm})\end{array}$ & $\operatorname{Vdd}(\mathrm{V})$ & $\begin{array}{c}\text { Pdd } \\
(\mathrm{mW})\end{array}$ \\
\hline This Work & $\begin{array}{c}0.25 \mu \mathrm{m} \mathrm{GaAs} \\
\text { pHEMT }\end{array}$ & $\begin{array}{l}\text { Casscode with dual } \\
\text { feedbacks and } \\
\text { BPDWB }\end{array}$ & $1-3.5 / 110 \%$ & $22.5-25.2$ & $0.5 \pm 0.05$ & $-20 \max$ & $9 \mathrm{~min}$ & $-3 \min$ & 5 & 330 \\
\hline Ref' $^{\prime} 1$ [25] & $\begin{array}{l}\text { GaAs pHEMT } \\
\text { Off the-shelf }\end{array}$ & $\begin{array}{l}\text { Folded-cascode with } \\
\text { intrinsic Cgd } \\
\text { feedback }\end{array}$ & $0.4-0.8 / 66.7 \%$ & 41 & $0.34 \max$ & $-8 \max$ & $\begin{array}{l}-25 \\
\min \end{array}$ & -32.8 & 5 & 406 \\
\hline $\operatorname{Ref}^{\prime} 02$ [26] & $\begin{array}{c}0.25 \mu \mathrm{m} \text { GaAs } \\
\text { pHEMT }\end{array}$ & Two-stage cascade & $2-4 / 66.7 \%$ & $32.1 \pm 0.44$ & $0.8-2.0$ & $-6 \max$ & NA & NA & 5 & 267 \\
\hline 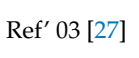 & $\begin{array}{l}0.5 \mu \mathrm{m} \mathrm{GaAs} \\
\text { pHEMT }\end{array}$ & $\begin{array}{l}\text { Casscode with } \\
\text { capacitive feedback }\end{array}$ & $1-4 / 120 \%$ & $20-23$ & $1.8-2.3$ & $-10 \max$ & NA & NA & 5 & 250 \\
\hline $\operatorname{Ref}^{\prime} 04$ [28] & $\begin{array}{c}0.25 \mu \mathrm{m} \mathrm{GaAs} \\
\text { pHEMT }\end{array}$ & $\begin{array}{l}\text { Casscode with } \\
\text { feedback }\end{array}$ & $1.65-2.75 / 50 \%$ & $19.7 \pm 1.5$ & $0.58 \max$ & NA & $10 *$ & NA & NA & NA \\
\hline Mod' 01 & GaAs pHEMT & HMC618ALP3E & $1.2-2.2 / 58.8 \%$ & 19 & 0.75 & -18 & 10 & -3 & 5 & 445 \\
\hline Mod' 02 & GaAs pHEMT & SKY67151-396LF & $0.7-3.8 / 130 \%$ & $19 @ 2.5 \mathrm{G}$ & $0.5 @ 2.5 \mathrm{G}$ & $-11 @ 2.5$ & $\begin{array}{c}17 \\
@ 2.5 \mathrm{G}\end{array}$ & $1.7 @ 2.5$ & 5 & 360 \\
\hline $\begin{array}{l}\text { Mod' } 03 \\
\text { Mod' } 04\end{array}$ & $\begin{array}{l}\text { GaAs pHEMT } \\
\text { GaAs pHEMT }\end{array}$ & $\begin{array}{l}\text { QPL9057 } \\
\text { TQL9093 }\end{array}$ & $\begin{array}{c}1.5-4 / 91 \% \\
0.6-4.2 / 150 \%\end{array}$ & $\begin{array}{l}18.5-21.7 \\
21.5-25\end{array}$ & $\begin{array}{l}0.5-0.85 \\
0.43-0.9\end{array}$ & $\begin{array}{l}-10 \max \\
-6 \max \end{array}$ & $\begin{array}{l}\text { NA } \\
\text { NA }\end{array}$ & $\begin{array}{l}\text { NA } \\
\text { NA }\end{array}$ & $\begin{array}{l}5 \\
5\end{array}$ & $\begin{array}{l}600 \\
250\end{array}$ \\
\hline
\end{tabular}

* Calculated from gain and OIP3.

From the comparison in the table, we can find that in the L-S frequency band, the LNA implemented in this paper within an octave bandwidth has better gain and NF performance than most of the LNAs compared.

\section{Conclusions}

In this paper, the proposed broadband LNA with the BPDWB network were designed. The theoretical analysis and graphical explanation of the BPDWB design were also discussed. By combining parasitic parameters and bias into BPDWB, the design efficiency and prediction accuracy of the matching circuit were greatly improved. Furthermore, thanks to dual feedbacks, the gain, bandwidth and linearity were also well compromised. The measurement results show that the LNA gain was greater than $22.5 \mathrm{~dB}$ and the noise figure less than $0.55 \mathrm{~dB}$ from $1 \mathrm{GHz}$ to $3.5 \mathrm{GHz}$, which verifies the good performance of the proposed LNA and the reliability of the proposed BPDWB network.

Author Contributions: Conceptualization, J.Z., F.W., H.Y., K.W., C.L., J.W., X.L. and Y.Y.; methodology, J.Z., X.L., J.W. and Y.Y.; software, J.Z., F.W. and H.Y.; validation, J.Z., S.Z. and K.W.; data curation, J.Z., F.W. and H.Y.; writing-original draft preparation, J.Z., S.Z. and K.W.; writing-review and editing, J.W., X.L., C.L. and Y.Y.; funding acquisition, X.L. All authors have read and agreed to the published version of the manuscript.

Funding: This research was funded by National Key R\&D Program, grant number E0G928C001. 
Conflicts of Interest: The authors declare no conflict of interest.

\section{References}

1. Conceição, F.; Gomes, M.; Silva, V.; Dinis, R.; Silva, A.; Castanheira, D. A Survey of Candidate Waveforms for beyond 5G Systems. Electronics 2021, 10, 21. [CrossRef]

2. Motz, C.; Paireder, T.; Pretl, H.; Huemer, M. A Survey on Self-Interference Cancellation in Mobile LTE-A/5G FDD Transceivers. IEEE Trans. Circuits Syst. II Express Briefs 2021, 68, 823-829. [CrossRef]

3. Ijiga, O.E.; Ogundile, O.O.; Familua, A.D.; Versfeld, D.J.J. Review of Channel Estimation for Candidate Waveforms of Next Generation Networks. Electronics 2019, 8, 956. [CrossRef]

4. Kukde, A.A.; Kumaravel, S.; Venkataramani, B. A high linearity folded cascode Low Noise Amplifier for wireless receivers. In Proceedings of the 2014 International Conference on Circuits, Power and Computing Technologies, Nagercoil, India, 20-21 March 2014; IEEE: Piscataway, NY, USA, 2015.

5. Laaouane, H.; Foshi, J.; Bri, S. Design of a low noise amplifier for LTE radio base station receivers. In Proceedings of the 2017 International Conference on Wireless Technologies, Embedded and Intelligent Systems (WITS), Fez, Morocco, 19-20 April 2017; IEEE: Piscataway, NY, USA, 2017.

6. Zhou, J.; Zhang, X.; Yu, Y.; Zhang, L.; Zhao, C.; Liu, H.; Kang, K. A 24-30 GHz CMOS LNA with 2.05 dB NF and 0.6 dB in-band gain ripple for 5G-applications. In Proceedings of the 2018 IEEE MTT-S International Wireless Symposium (IWS), Chengdu, China, 6-10 May 2018; IEEE: Piscataway, NY, USA, 2018; pp. 1-3.

7. Arsalan, M.; Wu, F. LNA design for future S band satellite navigation and 4G LTE applications. Comput. Modeling Eng. Sci. 2019, 119, 249-261. [CrossRef]

8. Mahmood, H.U.; Utomo, D.R.; Han, S.-K.; Kim, J.; Lee, S.-G. A Ku-Band RF Front-End Employing Broadband Impedance Matching with $3.5 \mathrm{~dB}$ NF and $21 \mathrm{~dB}$ Conversion Gain in 45-nm CMOS Technology. Electronics 2020, 9, 539. [CrossRef]

9. Nikandish, G.; Medi, A. Design and analysis of broadband darlington amplifiers with bandwidth enhancement in GaAs pHEMT technology. IEEE Trans. Microw. Theory Tech. 2014, 62, 1705-1715. [CrossRef]

10. Chien, K.H.; Chiou, H.K. A 0.6-6.2 GHz wideband LNA using resistive feedback and gate inductive peaking techniques for multiple standards application. In Proceedings of the 2013 Asia-Pacific Microwave Conference Proceedings (APMC), Seoul, Korea, 5-8 November 2013; IEEE: Piscataway, NY, USA, 2014.

11. Shekhar, S.; Walling, J.S.; Allstot, D. Bandwidth extension techniques for CMOS amplifiers. IEEE J. Solid-State Circuits 2006, 41, 2424-2439. [CrossRef]

12. Zailer, E.; Belostotski, L.; Plume, R. Wideband LNA Noise Matching. IEEE Solid-State Circuits Lett. 2020, 3, 62-65. [CrossRef]

13. Ali, M.; Hamed, H.F.A.; Fahmy, G.A. Small Group Delay Variation and High Efficiency 3.1-10.6 GHz CMOS Power Amplifier for UWB Systems. Electronics 2022, 11, 328. [CrossRef]

14. Zhang, S.; Wan, J.; Zhao, J.; Yang, Z.; Yan, Y.; Liang, X. Design of a Broadband MMIC Driver Amplifier with Enhanced Feedback and Temperature Compensation Technique. Electronics 2022, 11, 498. [CrossRef]

15. Vostokov, N.V.; Koblov, E.A.; Korolyov, S.A.; Revin, M.V.; Shashkin, V.I. Study of Electrophysical Characteristics of pHEMT Heterostructures by the Methods of Impedance Spectroscopy. IEEE Trans. Electron Devices 2018, 65, 1327-1332. [CrossRef]

16. Wang, S. The latest research progress of THz InP HEMT and HBT technology. Micro-Nano Electron. Technol. 2018, 55, 8.

17. Safonov, D.A.; Klochkov, A.N.; Vinichenko, A.N.; Sibirmovsky, Y.D.; Kargin, N.I.; Vasil'evskii, I.S. Electron effective masses, nonparabolicity and scattering times in one side delta-doped PHEMT AlGaAs/InGaAs/GaAs quantum wells at high electron density limit. Phys. E Low-Dimens. Syst. Nanostructures 2021, 133, 114787. [CrossRef]

18. Bakkali, M.E.; Elkhaldi, S.; Elftouh, H.; Touhami, N.A. Small-Signal Modeling of GaAs-pHEMT Using Direct Extraction Method. In Proceedings of the 2020 International Conference on Intelligent Systems and Computer Vision (ISCV), Fez, Morocco, 9-11 June 2020; IEEE: Piscataway, NY, USA, 2020.

19. Kim, C.W.; Jung, M.S.; Lee, S.G. Ultra-wideband CMOS low noise amplifier. Electron. Lett. 2005, 41, 384-385. [CrossRef]

20. Zhang, H.; Fan, X.; Sinencio, E.S. A Low-Power, Linearized, Ultra-Wideband LNA Design Technique. IEEE J. Solid-State Circuits 2009, 44, 320-330. [CrossRef]

21. Shahrabadi, S. Ultrawideband LNA 1960-2019: Review. IET Circuits Devices Syst. 2021, 15, 697-727. [CrossRef]

22. Rao, G.B. A high gain and high linear LNA for low power receiver front-end applications. In Proceedings of the 2016 International Conference on Communication and Signal Processing (ICCSP), Melmaruvathur, India, 6-8 April 2016; IEEE: Piscataway, NY, USA, 2016

23. Harsoori, M.M.; Zulkifli, T.; Abbas, U.; Sattar, S. A gain boosting single stage cascode LNA for millimeter-wave applications. In Proceedings of the 2017 IEEE Asia Pacific Conference on Postgraduate Research in Microelectronics and Electronics (PrimeAsia), Kuala Lumpur, Malaysia, 31 October-2 November 2017; IEEE: Piscataway, NY, USA, 2017.

24. BehzadRazavi, R. RF Microelectronics; Electronic Industry Press: Beijing, China, 2012.

25. Kulatunga, T.; Belostot Sk, I.L.; Haslett, J.W. 400-to-800-MHz GaAs pHEMT-Based Wideband LNA for Radio-Astronomy Antenna-Array Feed. IEEE Microw. Wirel. Compon. Lett. 2018, 28, 909-911. [CrossRef]

26. Nikandish, G.; Yousefi, A.; Kalantari, M. A Broadband Multistage LNA With Bandwidth and Linearity Enhancement. IEEE Microw. Wirel. Compon. Lett. 2016, 26, 834-836. [CrossRef] 
27. Song, H.; Shu, Y.; Guo, Y.; Hu, S. A 1-4 GHz low noise amplifier in 0.5- $\mu$ m E-Mode InGaAs pHEMT technology. In Proceedings of the 2017 7th IEEE International Symposium on Microwave, Antenna, Propagation, and EMC Technologies (MAPE), Xi'an, China, 24-27 October 2017; IEEE: Piscataway, NY, USA, 2017.

28. Ryu, K.K.; Kim, Y.H.; Kim, S.C. Low Noise and High Linearity GaAs LNA MMIC with Novel Active Bias Circuit for LTE Applications. J. Inf. Commun. Converg. Eng. 2017, 15, 112-116. 